\title{
François-Xavier Garneau: aspects bibliographiques
}

\author{
Paul Wyczynski
}

\section{François-Xavier Garneau: Homme}

SITUER LE CAS DE GARNEAU DANS LA PERSPECTIVE BIBLIOGRAPHIQUE $\mathrm{n}^{\prime}$ est point chose facile. A part de récents articles que contiennent le Dictionnaire pratique des auteurs québécois, le Dictionnaire des oeuvres littéraires du Québec et le Dictionnaire biographique du Canada (vol. Ix), rien ne nous paraît valable sur le côté strictement bibliographique de la vie et de l'oeuvre de Garneau. Il s'agit donc dans mon propos de montrer qui est F.-X. Garneau et comment se présentent les connaissances sur sa vie et sur son oeuvre. Mon exposé est suivi d'une bibliographie de l'oeuvre de Garneau et d'une note bibliographique sur les études qui lui ont été consacrées.

La critique d'autrefois et la critique d'aujourd'hui ont reconnu en FrançoisXavier Garneau 1'homme-clef du xıxe sièc' . adien-français. A la fois poète, historien, mémorialiste, aussi journaliste et traducteur à l'occasion, notaire de formation et greffier de la ville de Ouébec par devoir bien plus que par plaisir, il incarne en lui plusieurs vocations parmi lesquelles celle d'historien s'est imposée à son destin. Face à son époque qui est celle de Papineau et de l'Acte d'Union, il se montra observateur minutieux et, à des moments opportuns, l'homme clalrvoyant chez qui le jugement sur la société faisait rarement défaut.

Par ses origines, il appartient à la classe des travailleurs. Son lointain ancêtre, Louis Garnault, vint du Poitou vers I659. Par la force des choses, ses aïeux se firent agriculteurs, en cultivant les champs à l'Ange-Gardien et au village de Saint-Augustin. C'est son père qui décida de déménager la famille à Québec pour y vivre tant bien que mal de la pratique de divers métiers: le nouveau citadin fut tour à tour voiturier, sellier, tavernier... Sans être pauvre, le père de l'écrivain n'avait aucun moyen d'offrir à son fils une instruction poussée, mais les circonstances favorables s'en chargèrent à sa place.

Allant d'une école à l'autre - écoles simples et souvent improvisées comme celles du 'bonhomme Parent' et de Joseph-François Perreault - le jeune Garneau trouve finalement un abri dans l'étude prestigieuse du protonotaire Archibald Campbell, un Québécois d'origine écossaise, mécène des beaux- 
arts et des lettres, membre de la Literary and Historical Society of Quebec, humaniste convaincu et propriétaire d'une imposante bibliothèque. C'est là que le jeune Garneau fait son apprentissage de notaire; c'est là aussi qu'il complète, par des lectures et des discussions, son éducation qui, somme toute, se résumait à quelques notions de grammaire, de mathématiques, de géographie, d'histoire et de religion. Il reçoit sa 'commission de notaire' le 2 I juin r8 30 et, un an après, ayant fait un stage annuel supplémentaire à l'étude de Campbell, Garneau est prêt à voler de ses propres ailes à l'âge de vingt et un ans: il était né le II juin I809.

La première étape de la vie de Garneau correspond donc à la période de son enfance et de sa jeunesse: période de formation professionnelle dans laquelle s'inscrit un voyage de trois semaines aux Etats-Unis, période marquée aussi de quelques rêves littéraires - poétiques surtout - dont il serait difficile $\mathrm{d}^{\prime}$ expliquer les circonstances et les orientations.

La deuxième étape de la vie de Garneau ne dure que deux ans; elle va du 20 juin $\mathrm{r} 83 \mathrm{I}$ au 30 juin I833: la double traversée de l'Atlantique, vingt mois passés à Londres comme secrétaire de Denis-Benjamin Viger, et deux séjours de trois semaines à Paris. Le jeune notaire se met à apprendre l'histoire, celles du Canada, de l'Angleterre et de la France, mais aussi celles de l'Irlande et de la Pologne. Il étudie et observe le fonctionnement des institutions britanniques; il suit de près la marche des idées littéraires; il s'intéresse à la poésie où un pseudo-classicisme se mêle au romantisme; il remarque surtout les poèmes

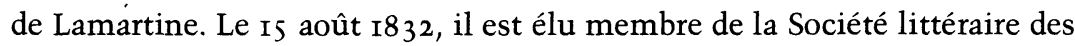
Amis de la Pologne. Il mûrit. Il apprend à penser et à écrire. Le 30 juin, via Liverpool, il revient à Québec plein de rêves et de projets, mais sans aucune idée précise sur son avenir. Il cherche sa place dans la société déjà fort bouleversée par les débats parlementaires et les querelles idéologiques, attiré par le journalisme, et taquinant la muse au grand plaisir de ses amis intimes.

La troisième période dans la vie de Garneau est la plus longue: elle s'étend de l'été I8 33 à l'hiver I866. Une trentaine d'années vouées à l'organisation de sa vie et de sa famille, non sans difficultés et surprises désagréables. La santé n'est pas toujours bonne. Comme notaire, Garneau est associé pendant un certain temps à Louis-Théodore Besserer, sans qu'on puisse parler ici de l'amorce d'une carrière. Occasionnellement, il est traducteur au parlement du Bas-Canada et, ensuite, pendant quelque temps, au parlement du CanadaUni, à Québec et à Kingston. Il tente sa chance dans le journalisme en fondant deux journaux éphémères: L'Abeille canadienne (7 déc. $1833-7$ fév. 1834 ) et L'Institut (7 mars I84I-22 mai I84I). Après la mort de ses parents (son père mourut le 7 août I832; sa mère, le 20 juillet 1835 ), il se marie, le 25 août 1835 , avec Marie-Esther Bilodeau, fille d'un cultivateur de la Canardière. Neuf enfants sont nés dont seulement quatre atteindront l'âge mûr: Alfred, 
Honoré, Eugène et Joséphine. Garneau cherche un emploi permanent; il le trouve finalement à l'Hôtel de ville de Québec où il devient greffier le Ier août I844. Il y restera jusqu'au ler mai 1864 .

Pendant tout ce temps, Garneau observe la vie sociale et politique. Ses études de notariat et son séjour à Londres lui ont procuré des connaissances plus larges que celles qu'avait alors un Québécois de son rang. Il savait aussi cultiver son esprit par des lectures choisies et la consultation constante des instruments de travail: encyclopédies, dictionnaires, guides de toutes sortes. En tant que citoyen du Bas-Canada, Garneau fut bouleversé par les événements de l'insurrection des années 1837-1838; il ne resta pas non plus insensible à l'Acte d'Union et aux retentissements qu'il avait provoqués. Il prit position contre la lutte violente et les interventions militaires lorsqu'il s'agissait de régler les problèmes politiques. Mais cela ne devait pas l'empêcher d'être un grand admirateur de Papineau. Quant à l'Union du Bas-Canada et du Haut-Canada, il y était farouchement opposé, au point d'en devenir exaspéré aux moments de la publication du Rapport Durham et de l'adoption de la loi au Parlement britannique. Il mettait ainsi ses connaissances à l'épreuve et son civisme au profit de la cause politique et idéologique de son pays. A sa mort, le 2 février I866, tout le Québec savait qu'un grand homme venait de mourir, un homme droit et lucide, un poète, un conteur et surtout un historien national. C'est lui, en effet, qui a donné à ses concitoyens une véritable Histoire du Canada pour réfuter les mensonges et faire éclater la vérité. L'ouvrage est de taille et sans doute le meilleur du xixe siècle, au point de vue littéraire, avec Les Anciens Canadiens de Philippe Aubert de Gaspé.

Le chercheur qui serait intéressé à la vie de Garneau devrait commencer par les études les plus récentes: il consultera donc d'abord l'esquisse biographique que j'ai publiée en 1977 , en collaboration avec Pierre Savard, dans le neuvième volume du Dictionnaire biographique du Canada (p. 327-336). Cette synthèse repose sur la recherche d'une dizaine d'années, rectifie les erreurs d'autrefois et s'attribue le mérite de présenter F.-X. Garneau dans une lumière neuve, puisée souvent à des sources jusqu'ici inconnues. En partie, cet article s'appuie sur les généalogie et chronologie de F.X. Garneau, publiées en 1968, en tête de mon édition critique du Voyage de Garneau. Une bonne vue d'ensemble sur la vie et l'oeuvre de Garneau est offerte aussi dans l'article paru dans le Dictionnaire pratique des auteurs québécois. Enfin, le catalogue François-Xavier Gameau, 1809-1866 que nous avons préparé, Pierre Savard et moi, pour une exposition Garneau qui se tint d'abord, en novembre I977, à la Bibliothèque nationale du Canada à Ottawa et, ensuite, en décembre 1978, au Centre culturel canadien, à Paris, peut être aussi une source de renseignements. Au cours des recherches sur F.X. Garneau que je poursuis 
depuis I960, j'ai consulté les registres d'état civil, les dépôts d'archives, la correspondance disponible de F.-X. Garneau et celle qui lui a été adressée, de même que la presse périodique de l'époque.

Parmi les premiers biographes de Garneau figurent l'abbé Henri-Raymond Casgrain, Pierre-Joseph-Olivier Chauveau et Louis-Michel Darveau. Le premier a publié chez Duquette, à Québec, au lendemain de la mort de Garneau, une biographie-témoignage qui est certes précieuse par l'abondance de détails mais qui manque de méthode rigoureuse. Même les citations doivent être scrupuleusement vérifiées. Ce livre, plusieurs fois réédité, fut longuement consulté par les professeurs et élèves comme source première sur le destin de l'historien national.

La deuxième source importante est l'ouvrage de Chauveau: FrançoisXavier Garneau, sa vie et ses oeuvres, intégré à tort, en $\mathrm{r} 882$, à la quatrième édition de l'Histoire du Canada. Chauveau a bien connu Garneau, mieux peut-être que Casgrain. L'esquisse biographique qui unit étroitement la vie à l'oeuvre littéraire est précieuse et, dans beaucoup de cas, unique. Je pense ici à certains détails concernant la famille Garneau, aux renseignements sur l'époque de l'historien et, surtout, au portrait physique et moral que l'écrivain a mérité de la plume de Chauveau. A ne pas oublier cependant que celui-ci, toujours au coeur de la bouillonnante activité politique, a dû composer hâtivement son ouvrage, et s'est trop fié à sa mémoire. Ainsi l'étude manque de précisions sur bien des périodes de la vie de Garneau. Le récit s'attarde dans bien des pages sur des généralités, des considérations qu'on qualifierait de digressions.

Entre les études de Casgrain et de Chauveau figure celle du notaire LouisMichel Darveau, publiée en I873 dans Nos hommes de lettres. Si Chauveau brosse de Garneau un portrait d'homme serein, bien que parfois pensif et un peu attristé, conforme à un certain idéal national des années I880, Darveau fait de lui la victime du clergé et de certains détracteurs des années I840. Par cet aspect, les vingt-cinq pages de Darveau apportent un témoignage intéressant que chaque biographe de Garneau doit prendre en considération, tout en le confrontant évidemment avec certaines confidences contenues dans les lettres de Papineau ou d'Alfred Garneau, autrefois inaccessibles aux chercheurs.

Il va de soi que la correspondance d'Alfred Garneau et celle d'Hector Garneau, respectivement fils et petit-fils de l'historien apportent à la connaissance de l'historien national plusieurs détails qui précisent la vie intime de l'écrivain. On trouvera aussi dans l'introduction de la cinquième édition (I9r3-I920), signée par Hector Garneau, une tentative de décrire, conformément à la tradition familiale, la vie de celui qui a assuré à la souche des Garneau de Saint-Augustin une renommée nationale. On ne pourrait se prononcer aujourd'hui non plus sur les derniers moments de l'historien national 
sans se référer à la lettre qu'Alfred Garneau adressa à Louis-Joseph Papineau le 20 janvier I869. Mais ni le fils ni le petit-fils n'ont écrit une biographie de leur ancêtre au sens vrai du mot. Leurs lettres, notes ou souvenirs n'apportent que quelques détails nouveaux à la vie et à l'oeuvre de leur illustre ancêtre.

En 1926, Gustave Lanctot publia à Toronto son François-Xavier Garneau; ce livre sera réimprimé, vingt ans plus tard, à Montréal, sous le titre Garneau, historien national. L'ouvrage marque un progrès dans la connaissance de Garneau, homme et écrivain, mais il est loin de satisfaire aux exigences d'une monographie rigoureuse. Il contribue surtout à maintenir le souvenir de Garneau qui, autour des années I945, lors du centenaire de l'Histoire du Canada, a connu une véritable renaissance. L'occasion a permis de réaliser un gros volume de 460 pages, Centenaire de l'Histoire du Canada de François-Xavier Garneau, dans lequel plusieurs spécialistes du passé canadien ont signé les études: Gustave Lanctot, Lionel Groulx, Olivier Maurault, Jean Bruchési, Armand Yon, Thomas Charland, Georges Robitaille, Maurice Hébert.

Un autre centenaire, celui de la mort de Garneau, a suscité la publication de l'ouvrage François-Xavier Gameau. Aspects littéraires de son oeuvre, aux Editions de l'Université d'Ottawa, en I966. Il s'agit là de quatre travaux de séminaire de mes étudiants de doctorat: Odette Condemine étudie le poète; François Gallays présente le journaliste; le père Charles Bolduc explique les métamorphoses de l'Hjstoire du Canada; la soeur Paul-du-Sauveur précise quelques particularités du discours historique. J'y publie moi-même un essai sur le Voyage de Garneau. Le volume vise fondamentalement à présenter Garneau-écrivain. Comme l'oeuvre ne se sépare pas de la vie, l'ouvrage renseigne sur l'oeuvre autant que sur la vie de Garneau.

Certes, à ces jalons marquants, il faudra ajouter un certain nombre d'articles de journaux et d'articles de revues dont la récente Bibliographie de la critique de la littérature québécoise dans les revues des XIXe et XXe siècles, compilée par Pierre Cantin, Normand Harrington et Jean-Paul Hudon, donne un bon aperçu (t. III, articles 8777-882I). Cependant, à l'examen de tous ces écrits et sans rien imputer à la bonne volonté de ceux qui les ont préparés et publiés, une constatation est à faire. On attend toujours une biographie de Garneau qui corrigerait les erreurs d'autrefois, synthétiserait les éléments biographiques dans un tout équilibré et révélerait la vraie physionomie physique et morale de l'homme et de l'écrivain. Le projet est de taille! Car Garneau domine son époque incontestablement. Pour le connaître, il faut non seulement étudier notre historien national mais tout le xıxe siècle. Alors, des ramifications politiques, sociales, littéraires et idéologiques nous mènent souvent au-delà du Canada: aux Etats-Unis, en France, en Angleterre ... C'est dans la totalité des problèmes divers et à l'aide des documents bien scrutés et bien interprétés qu'on arrivera un jour à situer l'homme dans son époque. 


\section{François-Xavier Garneau: Ecrivain}

L'oeuvre de Garneau comprend six catégories d'écrits: poésies, histoire, récit de voyage, journaux et articles de journaux, correspondance et avis de greffier. Inutile de dire qu'une bibliographie critique rédigée dans cette perspective nous rendrait un immense service. Elle est présentement en chantier et $j$ 'en tire, pour la présente étude, plusieurs renseignements importants.

\section{POÉSIES}

Il nous reste de Garneau vingt-sept poèmes. Le premier, La Coupe, date de juillet I830; le dernier, Le Vieux Chêne, fut publié dans Le Canadien du 29 septembre I84r. Pendant une dizaine d'années Garneau est uniquement connu comme poète. Il se veut romantique à la façon de Lamartine. A-t-il commencé à écrire des vers avant 1830 ? C'est fort possible, mais il n'en reste aucune trace. A-t-il écrit d'autres vers après I84I? J'en doute. Mais il se peut que ses vers non signés dorment encore dans quelques vieux journaux. Par exemple, P.-J.-O. Chauveau voudrait bien lui attribuer Le Voltigeur de retour et Le Chant du vieillard sur l'étranger, parus dans Le Canadien, les 2 et I6 juillet $183 \mathrm{I}$.

J'ai signé, dans le premier volume du Dictionnaire des oeuvres littéraires du Québec (p. 582-585), un article dans lequel j'étudie la genèse, la thématique et la forme des poèmes de Garneau. Je crois sans fausse prétention que c'est là la première présentation du monde poétique de l'auteur connu surtout comme historien. A la fin du présent travail, un choix d'études sur la poésie de Garneau permet de voir globalement ce qu'on a écrit sur le sujet depuis l'essai de Pierre-Joseph-Olivier Chauveau jusqu'à l'ouvrage de Jeanne d'Arc Lortie, La Poésie nationaliste au Canada français (1606-1867).

Dans le passé, la seule étude qui se découpe nettement des essais toujours trop généraux consacrés à Garneau au xixe siècle est celle de Chauveau: Etude sur les commencements de la poésie française au Canada et en particulier sur les poésies de M. F.-X. Garneau, publiée dans les Mémoires de la Société royale du Canada pour les années 1882 et 1883 . Le texte fut d'abord conçu comme discours lu à la Société royale du Canada, le 26 mai r883. Le contenu de l'article est le même que celui qu'on trouve dans le volume de Chauveau, François-Xavier Garneau, sa vie et ses oeuvres (Montréal, Beauchemin et Valois, 1883, 14, cccxciii p.). A l'époque, l'article de Chauveau plaisait. Aujourd'hui, il se révèle très peu utile pour un bibliographe soucieux d'exactitude. La première tentative valable pour connaître la poésie de Garneau selon les normes bibliographiques modernes me paraît être - et il faut bien le dire car je ne peux pas faire autrement - la bibliographie qui figure en tête de mon édition critique du Voyage (1968), p. 31-35 ... Lors d'un cours de doctorat, Odette Condemine en a dressé une liste qui figure au bas des pages de son article F.X. Garneau - poète: cette étude fait partie du volume François- 
Xavier Garneau. Aspects littéraires de son oeuvre. Vérifiée, ma bibliographie des poésies de Garneau paraît dans le Dictionnaire pratique des auteurs québécois (p. 276), aussi dans le Dictionnaire des oeuvres littéraires du Québec (p. 585 ). Tout récemment, John Hare a donné à Garneau une place de choix dans son Anthologie de la poésie québécoise du XIXe siècle, 1790-1890 (p. 87-II2), sans viser cependant à une bibliographie du sujet.

Voilà la perspective bibliographique de la poésie de Garneau. Les quinze dernières années ont fortement avancé les connaissances sur le sujet, sans $q^{\prime}$ 'on puisse cependant parler d'un aboutissement $\mathrm{qu}^{\prime}$ 'on souhaiterait voir dans une édition critique des textes poétiques en question.

\section{HISTOIRE DU CANADA}

On voit dans l'Histoire du Canada de Garneau l'un des ouvrages les plus prestigieux de la production littéraire du xixe siècle canadien-français. Elle fut conçue par un auteur profondément engagé dans la réflexion critique sur le sort du Bas-Canada. Agé de trente-trois ans, Garneau en publie le premier volume en I845; deux autres suivent en 1846 et en I848. Il cisèle cet ouvrage jusqu'à la fin de ses jours. L'Histoire du Canada est donc le résultat d'une réflexion progressive, d'un travail ardu; elle est animée d'un esprit critique et d'une vision qui projette dans la relation historique - surtout dans le 'Discours préliminaire' - une conception socio-politique de la nation, ce qui rehausse grandement, en plein milieu du xixe siècle, la reconstruction du passé canadien.

Pour un critique et un bibliographe l'Histoire du Canada n'est pas un sujet facile. L'ouvrage tient à la fois de l'histoire et de la littérature et, dans son cheminement chez les éditeurs, aboutit aujourd'hui à une neuvième édition qui n'est pas la meilleure. Il faut donc, en premier lieu, réfléchir sur les métamorphoses du texte et sur ce que les bibliographes appellent le 'texte de base'.

La vie de l'Histoire du Canada pourrait être divisée en trois étapes: les trois premières éditions sont l'oeuvre de l'auteur; les cinq éditions subséquentes ont été préparées par ses descendants, son fils Alfred et son petit-fils Hector; la neuvième met dans le copyright le nom de Françoiś Beauval et voit le jour grâce à l'initiative des Amis de l'Histoire. Soulignons dès maintenant, que pour nous le texte de base ne pourrait être que celui de la troisième édition (I8 59), la dernière du vivant de l'auteur.

La première édition compte quatre volumes: le premier parut en 1845 , le deuxième en $\mathrm{I} 846$, le troisième en $\mathrm{I} 848$ et le quatrième en $\mathrm{I} 852$. Les deux autres éditions, celles de 1852 et 1859 se composent chacune de trois volumes. La première question qui se pose est celle-ci: pourquoi la première édition regroupe-t-elle la matière en quatre volumes et pourquoi la publication du quatrième volume n'eut-elle lieu qu'en 1852 ? Ce détail mérite d'être 
clairement expliqué car il brouille souvent la mémoire des étudiants et la vue des professeurs et rend ardu le travail des bibliographes. Il faut retenir qu'initialement la relation historique de Garneau allait des origines du Canada jusqu'à la constitution de I79r. La grande fresque est divisée en douze livres et chaque livre se compose d'un certain nombre de chapitres. Or, dans la deuxième édition, l'historien décide de prolonger son récit jusqu'à l'Acte d'Union ( 1840 ), événement qui lui semble une énorme catastrophe pour la nation canadienne-française. Pour ce faire, Garneau rédige donc quatre autres livres qui relatent la matière de I79I à I84I. Les lecteurs de Garneau qui s'étaient procuré la première édition insistèrent auprès de l'auteur pour avoir la dernière partie de son récit sans être obligés d'acheter la deuxième édition en entier. Garneau fit donc faire un tiré à part des quatre derniers livres de la deuxième édition de son Histoire du Canada, qui devint en fait un 'supplément' pour les acheteurs de la première édition de son ouvrage. Et voilà pourquoi la première édition de l'Histoire du Canada figure sur les rayons des bibliothèques tantôt comme un ouvrage en trois volumes, tantôt en quatre.

Ma deuxième constatation concerne les changements d'éditeurs: dans le cas de la publication de l'Histoire du Canada, les deux premiers volumes furent publiés chez Napoléon Aubin, à Québec, le troisième, chez Fréchette et frère, également à Québec; la deuxième édition passe aux éditions John Lovell, à Montréal; la troisième édition est assumée par P. Lamoureux, à Québec. D'habitude on ne change pas d'éditeur si les affaires d'impression et de vente marchent bien. Or, dans le cas de Garneau, la publication de l'Histoire du Canada ne semble pas être une réussite de librairie. Bien plus! Garneau lui-même a dû participer financièrement à la mise en oeuvre de ses éditions, en essuyant des pertes dont il serait difficile de préciser aujourd'hui le montant. Traducteur adjoint à l'Assemblée législative à Kingston, Garneau fit certains arrangements, en novembre 1843 , pour la vente de son ouvrage: 'J'ai fait courir,' écrit-il dans sa lettre du I3 novembre I843 à son frère DavidBénoni, 'une petite liste des souscripteurs pour mon histoire du Canada et $j$ 'espère avoir une [cinquantaine] de souscripteurs: j'en ai déjà 40 parmi les plus notables des deux chambres'. Napoléon Aubin, journaliste fantaisiste, ne se vit pas confier le troisième volume de la première édition: même si les vraies raisons ne sont pas connues, on peut supposer que Garneau n'était pas satisfait du travail de Napoléon Aubin.

La deuxième édition de l'Histoire du Canada fut donc confiée à un éditeur plus expérimenté: John Lovell. Garneau caresse le rêve de vendre son ouvrage en France. Il envoie donc, en I8 52, à la Librairie Hector Bossange de Paris, I05 exemplaires de son livre dont le prix est fixé à I2 francs. L'état de compte établi par cette maison, le 3 I décembre 1853 , prouve que seulement 26 exemplaires de l'Histoire du Canada ont été vendus au pays de Lamartine à qui on adresse un exemplaire gratuit de même qu'à Jean-Jacques Ampère et à Napo- 
éon III. Les redevances ne suffisent même pas à payer les frais d'entrée, de transport et de publicité. A ce moment Garneau a probablement compris que son rêve de publier son Histoire du Canada en France était irréalisable. Certains lecteurs croyaient que l'ouvrage se vendait trop cher: le prix de la deuxième édition en trois volumes fut fixé à $22 \mathrm{~s} .6 \mathrm{p}$. et celui du 'volumesupplément' à $7 \mathrm{~s} .6 \mathrm{p}$.; en France le livre se vendait toujours I2 francs.

Si la troisième édition de l'Histoire $d u$ Canada passe à l'éditeur $\mathrm{P}$. Lamoureux, c'est que depuis déjà un certain temps John Lovell, qui détient les droits de la deuxième édition, est en pourparlers avec Andrew Bell pour une 'traduction libre' ou une 'adaptation anglaise' du texte français de Garneau. Celui-ci proteste énergiquement et exige publiquement 'une traduction fidèle et correcte' (Le Journal de Québec, I5 octobre 1859; Le Courrier du Canada, I9 octobre I859). Destiné d'abord à Lovell, le texte de la troisième édition paraît en I859, chez Lamoureux, à Québec. L'éditeur Lovell s'approprie cependant le droit de traduction et publie, en I860, History of Canada from the Time of its Discovery till the Union Year by Andrew Bell; une édition corrigée paraîtra en 1862 . La troisième édition de cet ouvrage fort controversé fut publiée à Montréal par Richard Worthington, et une autre par Belford Brothers, à Toronto. Il reste que la troisième édition de l'Histoire du Canada de Garneau, librement traduite par Andrew Bell, d'esprit anglais bien plus que canadien si l'on en juge par sa conférence surWolfe, de même que les attaques farouches de Maximilien Bibaud, contribuèrent certainement à l'état dépressif de l'historien national dans lesannées 1860 .

Il est incohtestable que Garneau a investi beaucoup d'argent et dans la préparation et dans la publication de son Histoire du Canada. Les deux petites subventions gouvernementales qu'il avait obtenues ne purent nullement équilibrer son budget. Il a admis dans sa lettre du 2I janvier à Chauveau que 'le besoin d'argent' l'a convaincu de préparer un 'abrégé' de l'Histoire $d u$ Canada qui mérita d'ailleurs, en I856, l'approbation de l'Eglise et se vendit très vite. Cet Abrégé parut d'abord en douze mille exemplaires, fut republié en 1858 et rapporta à Garneau une petite fortune qui compensa ses investissements déficitaires occasionnés par la publication de l'Histoire du Canada.

Alfred Garneau, le fils aîné de l'historien, a préparé la quatrième édition de l'Histoire du Canada. Au texte de son père il ajoute de nombreuses notes et corrections sans qu'on puisse les justifier. Le contrat avec la maison Beauchemin et Valois fut signé en septembre i879. Mais le retard de trois ans dans la publication de la quatrième édition est dû au fait que Chauveau, chargé de la biographie de Garneau devant constituer une partie intégrante de l'ouvrage, ne parvint pas à rédiger son manuscrit à la date prévue. C'est pour cette raison que la quatrième édition de l'Histoire du Canada (qui a pour texte de base l'édition de F.X. Garneau de 1859 / ne sort de presse qu'au début de 1883 , portant cependant, comme date de publication, l'année i882. Et le volume de 
Chauveau, considéré à la fois comme quatrième volume de cette édition et comme volume à part, contient une pagination en chiffres romains: I4cclxxxii pages. Tout fait croire que le volume de Chauveau fut initialement conçu comme l'introduction à la quatrième édition de l'Histoire du Canada.

Ce que le petit-fils de l'historien national, Hector Garneau, a apporté à l'ouvrage de son père se trouve dans trois éditions de l'Histoire du Canada, publiées entre deux centenaires: celui de la naissance de F.-X. Garneau (I909) et celui de l'Histoire du Canada (I945). Erudit remarquable, Hector Garneau reprend à sa façon la quatrième édition et en publie une cinquième, avec des appendices, une introduction substantielle et une préface de Gabriel Hanotaux, membre de l'Académie française. L'ouvrage aurait dû paraître chez Alcan, à Paris, en deux volumes. Mais la guerre brouilla les plans. C'est ainsi que le premier volume parut en I9I 3 et le deuxième, en I920. Il est donc facile d'imaginer pourquoi les deux éditions suivantes, publiées chez le même éditeur, en 1920 et en 1928 , ne sont à vrai dire que des réimpressions de la cinquième.

A l'occasion du centenaire de l'Histoire du Canada, aidé de quelques amis, Hector Garneau publie, entre 1944 et I946, une 'histoire expurgée' de son père. On veut rendre l'ouvrage 'abordable' au lecteur. On supprime donc la 'Preface' d'Hanotaux, le 'Discours préliminaire', les notes critiques, les passages peu 'catholiques' ... Il faut que le texte soit conforme à 'la foi ancestrale', comme l'explique Hector Garneau dans son article Nouvelle Introduction à l'Histoire du Canada, publiée en mars I9I3. Le texte de F.-X. Garneau est regroupé maintenant en huit volumes; le neuvième est un index. A tout prendre, l'entreprise familiale améliore peu le fruit du travail de l'historien national.

Enfin, en 1969, les Amis de l'Histoire (copyright: François Beauval, Paris) publient une Histoire du Canada en six volumes, joliment illustrée mais sans aucune note ni introduction. Il faut faire quelques comparaisons pour s'apercevoir que les 'Amis de l'histoire' utilisent le texte de la quatrième édition en modifiant, on ne sait pour quelle raison, le titre de Garneau qui devient: Histoire du Canada français. Une pareille chose est inadmissible.

Voilà les métamorphoses fondamentales de l'Histoire du Canada de Garneau. Il nous faut maintenant parler des sources et des études qui s'y rattachent. Presque tous ceux qui s'en occupent analysent l'ouvrage sous un angle particulier. Casgrain, Darveau, Chauveau, les trois biographes contemporains de Garneau, parlent dans leurs études de l'Histoire du Canada. Une bonne partie des articles qui traitent des trois premières éditions de l'Histoire $d u$ Canada se trouvent réunis dans la thèse de maîtrise de la soeur Georgette Barette, m.i.c., 'Garneau et la critique', soutenue à l'Université d'Ottawa en 1968. Parmi les critiques les plus récentes, je nommerais Guy Frégault (L'Actualité de Garneau, Action universitaire, mars I945), Louis Philippe 
Saint-Martin (L'Histoire du Canada de F.-X. Garneau et la critique, Revue d'Histoire de l'Amérique française, juin 1954) et le R.P. Charles Bolduc (Métamorphoses de l'Histoire du Canada, François-Xavier Garneau, aspects littéraires de son oeuvre). Sur l'ensemble de la question, on lira l'article que Pierre Savard et moi avons publié dans le Dictionnaire des oeuvres littéraires $d u$ Québec (vol. 1, p. 347-355). Pierre Savard a également publiée une étude bien documentée sur les rééditions de l'Histoire du Canada dans la Revue d'Histoire de l'Amérique française (vol. 28, $\mathrm{n}^{0} 4$, mars I975, p. 539-553).

VOYAGE EN ANGLETERRE ET EN FRANCE

DANS LES ANNÉES I83I, I832 ET I 833

Ce récit parut d'abord en tranches dans le Journal de Québec entre le i8 novembre 1854 et le 29 mai I 855 . Publié en volume quelques mois après, chez Augustin Côté, il fut détruit presque aussitôt, l'auteur étant insatisfait de la présentation du volume. A peine dix exemplaires furent sauvés de l'autodafé. Sensiblement abrégé, le texte parut en I863 dans le collectif La Littérature canadienne de 1850 à 1860; ce même texte parut en volume, en I878, avec ce titre fautif donné par Casgrain: 'Voyages'.

Récit intéressant du séjour de deux ans en Europe, ce texte est probablement le mieux étudié parmi les récits de Garneau. J'ai publié, en I968, aux Editions de l'Université d'Ottawa, l'édition critique du Voyage avec une longue introduction qui présente et éclaire de plusieurs façons la relation de Garneau. Outre les notes et variantes, le lecteur y trouve une 'Généalogie et chronologie de F.-X. Garneau' et une 'Bibliographie' concernant le Voyage autant que l'oeuvre entière de Garneau. Nous disposons donc d'un instrument de travail qui, sans être définitif, permet aux intéressés de se familiariser facilement avec la vie et les écrits de Garneau.

LES JOURNAUX DE F.-X. GARNEAU ET SA CONTRIBUTION

À LA PRESSE PÉRIODIQUE

Garneau a fondé et rédigé deux hebdomadaires: L'Abeille canadienne et L'Institut dont le premier titre fut L'Institut ou Journal des étudiants. Ces deux feuilles ne durèrent que très peu de temps: la première, du 7 décèmbre $1833 \mathrm{au}$ 8 février 1834 ; la deuxième, du 7 mars $184 \mathrm{I}$ au $2 \mathrm{I}$ mai I84I. Leurs présentation et style n'étaient pas sans relation avec The Penny Magazine publié par Charles Knight à Londres à partir du 3I mars I832, ou avec Le Magazine pittoresque de Paris: il s'agissait d'informer, d'instruire et de distraire le lecteur moyen. D'habitude, les articles n'y sont pas signés. Il serait donc difficile de préciser la part exacte d'écriture venant dans les deux cas de la plume de Garneau. Ce qu'on peut dire, cependant, c'est qu'une bonne partie de ces deux journaux provient de lui. J'ai étudié cette question lors d'un cours de doctorat, professé en 1964; l'un des étudiants, François Gallays, a alors préparé un travail de séminaire dont le texte fut publié dans François-Xavier Gameau. Aspects 
littéraires de son oeuvre, sous le titre F.X. Garneau et le journalisme (p. 45-63). Nous possédons donc des renseignements précis sur les préoccupations journalistiques de Garneau.

Dans les journaux de l'époque Garneau a publié un certain nombre d'articles, avec ou sans signature, parfois seulement paraphés de ses initiales. Le relevé de ces écrits n'a pas encore été publié et je doute qu'il soit jamais complet. Nous possédons cependant dans nos dossiers plusieurs de ces articles, de valeur inégale, certes, mais souvent révélateurs au point de vue des idées de Garneau et de sa participation à la vie sociale, littéraire et politique.

\section{CORRESPONDANCE}

La correspondance est toujours d'une grande utilité pour connaître la vie et l'oeuvre d'un écrivain. Souvent elle éclaire son destin de renseignements précieux qui, ajoutés aux autres faits biographiques, permettent de voir mieux dans le monde intime de l'écrivain. Or, dans le cas de Garneau, jusqu'en 1970, la situation sous cet angle paraissait quasi désespérée: le biographe avait à sa disposition à peine quelques lettres de Garneau et quelques fragments cités ici et là dans les études. Dans la reconstruction de la vie et dans l'interprétation de l'oeuvre de Garneau, la recherche était, par conséquent, peu commode chaque fois qu'il fallait confronter certains faits établis ou certaines propositions d'interprétation avec les intentions nettement exprimées par l'auteur. La situation a cependant changé depuis qu'Edouard Garneau, arrière petit-fils de l'historien, a versé au CRCCF le Fonds des Garneau. Marc Lebel, chargé d'établir l'édition critique de la correspondance de Garneau, a dressé en 1978 une liste des lettres de F.X. Garneau et l'a publiée dans le Bulletin du CRCCF: il s'agit d'un ensemble de 152 pièces. Certaines lettres furent adressées à des destinataires au nom prestigieux: Louis-Joseph Papineau, Jacques Viger, Edmund Bailey O'Callaghan, Pierre-Joseph-Olivier Chauveau, Louis-Hippolyte LaFontaine, Xavier Marmier, Georges-Barthélemy Faribault, Jean-Jacques Ampère, Henri-Emile Chevalier, Pierre Margry, Edouard Bossange, Etienne Parent, Hector-Louis Langevin, Edme Rameau de Saint-Père, Henri Martin, Henri-Raymond Casgrain, John Lovell ...

La correspondance réunie dans le cadre du Projet d'édition critique de F.X. Garneau, dirigé par Pierre Savard et moi, comprend aussi cent trente-neuf lettres adressées à $\mathrm{l}^{\prime}$ historien national. C'est de là aussi que vient un éclairage intéressant. Bref, les lettres de Garneau et les lettres à Garneau élargissent considérablement les connaissances sur l'homme qui, très longtemps, n'ont dépendu que des études de Casgrain, de Chauveau et de Lanctot.

\section{AVIS DE GREFFIER}

Pendant vingt ans Garneau fut greffier de la ville de Québec. Pendant vingt ans, il assista aux réunions du Conseil de ville et en rédigea les procès-verbaux. Tout ce que la ville de Québec lui demandait d'enregistrer en anglais et 
en français appartient évidemment aux dossiers de la Corporation de la ville de Québec. Une partie de ces écrits consiste dans les 'avis' publiés dans les journaux québécois et montréalais de l'époque traitant de sujets fort divers qui concernent la ville de Québec. Ces 'avis' n'ont pas de valeur littéraire. Conçus sous forme d'annonces, ils communiquent au grand public les travaux en marche, les projets pour lesquels on cherche des créditeurs. Garneau a rédigé quelque quatre cents 'avis'.

\section{L'Edition critique Garneau dans la perspective bibliographique}

Les recherches qu'on nomme aujourd'hui 'Projet Garneau' comprennent une série de travaux dont l'origine remonte aux années 1960. Pendant les dix premières années, $j$ 'ai travaillé seul, sans subvention ni plan établi. A cette époque, j'ai fait des recherches sur la vie de Garneau et sur certains aspects de son oeuvre: il en est résulté, en 1968, une édition critique du Voyage en Angleterre et en France dans les années 1831, 1832 et 1833 de F.-X. Garneau. J'ai professé à la même époque, de 1963 à 1966, un cours de doctorat sur F.-X. Garneau qui eut comme suite, aux Editions de l'Université d'Ottawa, en I966, la publication d'un collectif, François-Xavier Garneau. Aspects littéraires de son oeuvre.

En I97I commence, pourrait-on dire, la deuxième étape de nos recherches sur Garneau: 1'historien Pierre Savard s'est joint au projet. Le Conseil des Arts du Canada nous a alors accordé une subvention pour préparer une 'Edition critique de l'Histoire du Canada', qui devint vite 'Edition critique des Oeuvres complètes de François-Xavier Garneau'. Avec l'aide d'assistants de recherche, nous avons entrepris des recherches de longue haleine: pour connaître Garneau, il faut scruter à fond tout le xixe siècle canadien-français. Les recherches ainsi conçues nous ont menés très loin. A fin de préparer les notes, il nous a fallu étudier les faits, les personnages et les lieux. Pour les variantes, il nous a fallu comparer les textes. Ici, l'Histoire du Canada pose un problème réel. Notre texte de base est l'édition de I859. Comparé aux deux premières éditions - faits, style, ponctuation - ce texte représente quelque vingt-deux mille variantes. La relation de Garneau exige de nombreux commentaires historiques et géographiques de même que de fréquentes précisions linguistiques. Nous croyons posséder l'essentiel de la matière pour rédiger et publier une édition critique des 'Oeuvres complètes de F.-X. Garneau'.

Selon le plan déjà établi, l'édition aura douze volumes:

vol. I. Chronologie et Album Garneau

vol. 2. Poésie

vol. 3. Voyage en Angleterre et en France dans les années 1831, 1832 et 1833

vol. 4. Correspondance

vol. 5. Ecrits divers

vol. 6-Ir. Histoire du Canada

vol. I2. Bibliographie critique et index 
Chaque volume comprendra, outre le texte établi, une introduction, un choix de bibliographie et un index. Il va de soi que les petites bibliographies sélectionées, propres à éclairer chaque volume en particulier, seront regroupées dans la Bibliographie critique qui constituera le dernier volume. Quant à la Chronologie, elle sera différente de ce qu'on voit en général dans une édition critique: nous la concevons comme une suite d'événements assez détaillés, constituant, en somme, une sorte de biographie en petites séquences dans lesquelles nous résumerons tout ce que nous savons sur la vie, l'oeuvre, le milieu et l'époque de F.-X. Garneau.

Ainsi se présente, me semble-t-il, la situation bibliographique de F.-X. Garneau. La bibliographie critique que nous envisageons présentera à peu près tous les écrits de Garneau et toutes les études, thèses, notes et annonces qui lui ont été consacrées. Mais pour ce faire, il faut investir beaucoup de temps et beaucoup de patience. On pourrait ici se rappeler le mot de Marie CurieSklodowska: travaillent vite ceux qui travaillent lentement, pourvu que le plan soit suivi et l'esprit inventif demeure actif partout dans l'oeuvre enterprise.

\section{Bibliographie}

\section{A. OEUVRE DE F.-X. GARNEAU}

I. Poésies

La Coupe

La Coupe, dans Le Canadien, vol. 3, $\mathrm{n}^{0} 7 \mathrm{I}, \mathrm{I} 8$ oct. 1833, p. 2. (Poème signé d'initiales 'F.X.G.' et daté de 'Québec, Juillet, $1830^{\prime}$ ').

Le Voltigeur, 1812

Le Voltigeur, dans Le Canadien, vol. I, $\mathrm{n}^{0}$ Io, 8 juin I83I, p. I (sans signature); reproduit également sans signature avec sous-titre -Voltigeur. Souvenirs de Châteauguay - dans Le Répertoire national de James Huston, Montréal, Imprimerie Lovell et Gibson, 1848, vol. I, p. 185-186; 2e ed., Montréal, Valois, 1893, vol. I, p. 222-223; avec la signature et le titre légèrement modifié - Le Voltigeur, 1812 - , le poème paraît dans La Nouvelle Lyre canadienne ou Chansonnier de tous les âges, Montréal, Zéphirin Chapeleau, 1858, p. I19-120; publié ensuite dans La Nouvelle Lyre canadienne, Montréal, Beauchemin, 1895, p. 33-34. (Air: 'Le Jeune Edmond allait ....'.)

Dithyrambe sur la mission de M. Viger, envoyé des Canadiens en Angleterre Dithyrambe sur la mission de M. Viger, envoyé des Canadiens en Angleterre, dans Le Canadien, vol. I, $\mathrm{n}^{0}$ 34, 3 I août I83I, p. r. (Poème non signé; mais une note insérée à la troisième page de la même livraison du Canadien attribue le texte à Garneau.)

La Liberté prophétisant sur l'avenir de la Pologne

La Liberté prophétisant sur l'avenir de la Pologne, dans Polonia (Londres), $\mathrm{n}^{0} 3$, oct. 1832, p. $185-187$.

Voyageur

Elégie. Par un jeune Canadien maintenant à Londres, dans Le Magasin du BasCanada, vol. 2, n ${ }^{0} 6$, livr. de déc. 1832, p. 225-229, (d'après une lettre manuscrite, contenant ce poème, écrite par Garneau à Londres et expédiée à son ami 
Pierre Winter); reproduit dans Le Canadien, vol. 2, $\mathrm{n}^{0}$ 129, rer mars I833, p. I; sous le titre de Voyageur, le poème paraît dans Le Répertoire national de James Huston, éd. 1848, vol. I, p. 20I-205, éd. 1893, vol. I, p. 239-243. (Le Magasin du Bas-Canada et Le Canadien ont reproduit le texte sans signature; dans les deux éditions du Répertoire national le poème est identifiée ainsi: 'F.-X. Garneau (Londres)'.)

\section{La Pologne}

Ode. Souvenirs d'un Polonais, dans Le Canadien, vol. 3, $\mathrm{n}^{0} 32$, 19 juillet 1833 , $\mathrm{p}$. I (le premier poème signé: 'F.X. Garneau' ${ }^{\prime 1}$ ); repris dans La Minerve, vol. $7, \mathrm{n}^{0}{ }_{48}$, 29 juillet $\mathrm{I} 833$, p. I; reproduit sous le titre La Pologne, dans Le Répertoire national de James Huston, éd. 1848, vol. I, p. 322-327, éd. I893, vol. I, p. 368 373. (Huston range à tort ce poème parmi les écrits de 1835 : il est certain que le texte a été rédigé par Garneau au cours de l'été de 1833 .)

La Harpe

La Harpe, dans Le Canadien, vol. 3, $\mathrm{n}^{0} 35,26$ juillet I833, p. I (signé); reproduit dans Le Répertoire national de James Huston, éd. I848, vol. I, p. 236-237, éd. I893, vol. I, p. 276-278. (Huston désigne à tort à ce poème une place parmi les écrits datant de 1834.$)$

Le Canadien en France

Le Canadien en France, dans Le Canadien, vol. 3, n ${ }^{\circ} 42, \mathrm{I} 2$ août $\mathrm{I} 833$, p. 2 (avec la signature de Garneau); reproduit dans La Minerve, vol. 7, $\mathrm{n}^{0} 53$, I5 août 1833 , p. I, aussi dans Le Répertoire national de James Huston, avec une note biographique sur l'auteur et la signature qui se lit ainsi: 'F.X. Garneau (Paris)', éd. I 848, vol. I, p. 199-200, éd. I893, vol. I, p. 237-238. (Air: 'Du Dieu de bonnes gens'.)

L'Etranger

L'Etranger, dans Le Canadien, vol. 3, n ${ }^{0} 50,30$ août I833, p. I /avec la signature de Garneau); reproduit dans La Minerve, vol. 7, $\mathrm{n}^{0} 59,5$ sept. I833, p. I; aussi dans Le Répertoire national de James Huston, éd. I848, vol. I, p. 222-223; éd. I893, vol. I, p. 262-263.

Châteauguay

Châteauguay, dans Le Canadien, vol. 3, n ${ }^{\circ} 53,6$ sept. 1833, p. I (signé); reproduit dans L'Echo du pays, vol. I, $\mathrm{n}^{0} 3$ I, 26 sept. I833, p. I.

L'An 1834

Le Premier Jour de l'An 1834, dans Le Canadien, vol. 3, $\mathrm{n}^{0}$ I03, 2 janv. I834, p. I (sans signature); reproduit dans La Minerve, vol. $7, \mathrm{n}^{0} 95,9$ janv. I834, p. I (sans titre ni signature); sous le titre $L^{\prime} A n 1834$, et avec la signature de Garneau, le texte paraît ans Le Répertoire national de James Huston, éd. I848, vol. I, p. 234, éd. I 893 , vol. I, p. 274-275, où l'on précise que l'air est celui de: 'Reinedu monde, ô France, ô ma patrie!'.

Pourquoi désespérer?

Chanson, dans Le Canadien, vol. 4, $\mathrm{n}^{0} 23$, 30 juin 1834, p. 2 (poème signé d'initiales 'F.X.G.'); reproduit dans La Minerve, Québec (du Canadien du 30) chanson, vol. $8, \mathrm{n}^{0} 4 \mathrm{I}$, 3 juillet $\mathrm{r} 834$, p. 2; sous le titre changé, Pourquoi désespérer?, le texte est publié dans Le Répertoire national de James Huston, éd. I848, vol. I, p. 235-236, éd. 1893, vol. I, p. 274-275. (Air: 'De la Sentinelle ....)

Le Marin

Le Marin canadien, dans Le Canadien, vol. 6, $\mathrm{n}^{0} 7,23$ mai 1836, p. I (signé

I. Cette forme de signature se trouve souvent utilisée par l'auteur à la fin de certains de ses poèmes. 
d'initiales 'F.X.G.'); sous le titre abrégé, Le Marin, à tort rangé parmi les textes de 1834, dans Le Répertoire national de James Huston, éd. I848, vol. I, p. 242243, éd. I893, vol. I, p. 283-284.

Le Tombeau d'milie

Le Tombeau d'Emilie, dans Le Canadien, vol. 6, $\mathrm{n}^{0} 3 \mathrm{I}$, 20 juillet $\mathrm{I} 836$, p. I (texte signé d'initiales 'F.X.G.').

Au Canada

Pourquoi mon âme est-elle triste?

Au Canada, dans Le Canadien, vol. 6, $\mathrm{n}^{0}$ I 18, Io févr. I837, p. I (signé d'initiales 'F.-X.-G.'); reproduit dans La Minerve, vol. II , n ${ }^{0}$ 5, 27 févr. I837, p. I; avec le sous-titre 'Pourquoi mon âme est-elle triste?', le texte paraît dans Le Répertoire national de James Huston, éd. r848, vol. 2, p. 30-33, éd. 1893, vol. 2, p. 48-5I; un extrait tiré de la première et de la troisième partie de ce poème, sous le titre Hymne de Jean-Baptiste à sa patrie. Au Canada, fut imprimé dans Le Journal de Québec, vol. 7, $\mathrm{n}^{0} 88,30$ juin I849, p. I, repris dans La Minerve, vol. 21, $\mathrm{n}^{0} 85,-2$ juillet 1849 , p. 2; 1'ex trait fut imprimé également comme une feuillet et jeté dans la foule, pendant le défilé de Saint-Jean-Baptiste, à Québec, le 24 juin I849. A Lord Durham

A Lord Durham, dans Le Canadien, vol. 8, $\mathrm{n}^{0} \mathrm{I} 5,8$ juin $\mathrm{I} 838$, p. I; reproduit dans Le Populaire, vol. $2, \mathrm{n}^{0} 25, \mathrm{I} 3$ juin $\mathrm{I} 838$, p. I. (Dans les deux cas le poème est signé d'initiales 'F.X.G..')

A mon fils

A mon fils, dans Le Canadien, vol. 8, $\mathrm{n}^{0} 49,27$ août 1838 , p. I (signé d'initiales 'F.X. $\mathrm{G}^{\prime}{ }^{2}$ avec mention 'inédit'); reproduit dans Le Répertoire national de James Huston, éd. I848, vol. 2, p. 77-78, éd. 1893, vol. 2, p. 98-99.

Le Rêve du soldat

Le Rêve du Soldat, dans Le Canadien, vol. 8, $\mathrm{n}^{0} 80,7$ nov. I838, p. I (signé d'initiales 'F.X.G.', et précédé d'une brève note explicative); sous le titre Le Rêve du soldat reproduit dans Le Répertoire national de James Huston, éd. 1848, vol. 2, p. 69-74, éd. 1893, vol. 2, p. 89-94.

La Presse

Poésie du jour de l'An, dans Le Canadien, vol. $8, \mathrm{n}^{0}$ Io2, 4 janv. I839, p. I (poème non signél; reproduit sous le titre La Presse, attribué à Garneau, dans Le Répertoire national de James Huston, éd. I848, vol. 2, p. Io9-III, éd. I893, vol. 2, p. I32-I34.

Les Oiseaux blancs

Les Oiseaux blancs, dans Le Canadien, vol. 8, $\mathrm{n}^{0}$ I37, 27 mars I839, p. I (signé d'initiales 'F.-X. G.'); publié aussi dans Le Répertoire national de James Huston, éd. I848, vol. 2, p. I24-I26, éd. I893, vol. 2, p. I48-I49; reproduit dans Journal de l'Instruction publique, vol. 8, $\mathrm{n}^{0} \mathrm{I} 2$, déc. 1864, p. 165; dans l'Alliance nationale, vol. $9, \mathbf{n}^{0} \mathrm{I}, \mathrm{1903}$, p. I; la première, la quatrième et la septième strophes sont reproduites dans Le Passe-Temps, $\mathrm{n}^{0} 464,4$ janv. I9I3, p. 5IO-5II, avec la mélodie de D.-A. Fontaine et l'accompagnement de Léon Ringuet.

Louise. Légende canadienne

Louise. Une légende canadienne, dans Le Canadien, vol. $9, \mathrm{n}^{0}$ I 10, 17 févr. 1840 , p. I (signé d'initiales 'F.X.G.'); reproduit dans Album littéraire et musical de la

2. C'est à la fin de ce poème et à la fin des Oiseaux blancs, que Garneau utilise un trait d'union entre les initiales de ses prénoms: 'F.-X. G.'. Partout ailleurs les initiales de l'auteur se lisent 'F.X.G.' 
Revue canadienne, vol. I, juillet I846, p. I60-I64; aussi, sous le titre légèrement modifié, Louise. Légende canadienne, publié dans Le Répertoire national de James Huston, éd. 1848, vol. 2, p. 168-178, éd. 1893, vol. 2, p. 193-203.

L'Hiver

L'Hiver, dans Le Canadien, vol. II $\mathrm{n}^{0} 29,29$ mai 1840 , p. 2 /signé d'initiales 'F.X.G.'); reproduit sans titre dans Journal de l'Instruction publique, vol. 2, $\mathrm{n}^{0}$ I2, déc. 1858, p. 205; paraît dans Le Répertoire national de James Huston, éd. I848, vol. 2, p. 138-140, éd. 1893, vol. 2, p. 163-165.

Le Dernier Huron

Le Dernier Huron, dans Le Canadien, vol. I I, $\mathrm{n}^{0} 39$, I2 août $\mathrm{I} 840$, p. I (avec une note et signé d'initiales 'F.X.G.'|; reproduit dans Album littéraire et musical de la Revue canadienne, vol. I, juin I846, p. I48-150; dans Le Répertoire national de James Huston, éd. I848, vol. 2, p. I47-I50, éd. I893, vol. 2, p. 172-175; paru' également dans L'Abeille (du Petit Séminaire de Québec), vol. 4, $\mathrm{n}^{0} 26,29$ avril I852, p. I; dans Le Journal de l'Instruction publique, vol. Io, $\mathrm{n}^{0} 2$, 1866, p. I7-I8.

Les Exilés

Les Exilés, dans L'Institut ou Journal des Etudiants, vol. I, $\mathrm{n}^{0} \mathrm{I}, 7$ mars $\mathrm{I} 84 \mathrm{I}, \mathrm{p}$. I (poème signé d'initiales 'F.X.G.'); dans Le Répertoire national de James Huston, éd. 1848, vol. 2, p. 220-222, éd. I893, vol. 2, p. 247-250.

Le Papillon

Le Papillon, dans Le Canadien, vol. I I , $\mathrm{n}^{0} 56$, I7 sept. I84I, p. I (signé d'initiales 'F.X.G.'); reproduit dans L'Aurore des Canada, vol. Io, $\mathrm{n}^{0} 74,28$ nov. I848, p. I; dans Le Répertoire national de James Huston, éd. 1848, vol. 2, p. 199-200, éd. I893, vol. 2, p. 225-226.

Le leune Exilé

Le leune Exilé, poème autographe de $\mathrm{F}$. X. Garneau, daté de $\mathrm{I} 84 \mathrm{I}$, composé de trois quatrains, encadrés d'octaves, écrit en octosyllabes. Fonds F.-X. G.

Le Vieux Chêne

Le Vieux Chêne, ${ }^{3}$ dans Le Canadien, vol. II , ${ }^{0}$ 6I, 29 sept. I84I, p. I (signé d'initiales 'F.X.G.'); reproduit dans Album littéraire et musical de la Revue canadienne, vol. I, sept. 1846, p. 224-225; aussi dans Le Répertoire national de James Huston, éd. 1848, vol. 2, p. 18I-184, éd. 1893, vol. 2, p. 206-209.

\section{Histoire}

I. Histoire du Canada

Ière édition -

Histoire du Canada depuis sa découverte jusqu'à nos jours, Québec, 4 tomes; t. I: Imprimerie de N[apoléon] Aubin, 1845, 558 p.; t. 2: 1846, 578 p.; t. 3:

Imprimerie de Fréchette et frère, 1848,566 p.; t. 4, Montréal, Imprimé par John Lovell, 1852,326 p. (Atpréciser que le 4e volume ne comprend que les quatres derniers livres de la seconde édition: ils relatent les événements de 1792 jusqu'à

3. D'après nos recherches, Garneau a publié vingt-sept poèmes dont Le Vieux Chêne est le demier. Il est possible que d'autres de ses poèmes se cachent dans des journaux des années I830, sous le couvert de l'anonymat. P.-J.-O. Chauveau est enclin à attribuer à Garneau deux autres pièces: Le Voltigeur de retour, publié dans $L e$ Canadien, vol. $\mathrm{I}, \mathrm{n}^{0} 17,2$ juillet $\mathrm{1} 83 \mathrm{I}$, p. $\mathrm{I}$, et $L e$ Chant du vieillard sur l'étranger, publié aussi dans Le Canadien, vol. I, $\mathrm{n}^{0} 2 \mathrm{I}$, I6 juillet I83I, p. I. Même si plusieurs arguments militent en faveur de la supposition de Chauveau, les preuves concluantes restent à trouver. 
1'Union, récit qui ne figure pas dans la première édition). 2e édition -

Histoire du Canada depuis sa découverte jusqu'à nos jours, Québec, Imprimé

par John Lovell, I852, 3 tomes; t. I: xxii, 377 p.; t. 2: 454 p.; t. 3: 4I2 p. 3e édition -

Histoire du Canada depuis sa découverte jusqu'à nos jours, Québec, Imprimé

par P. Lamoureux, 1859, 3 tomes; t. I: xxii, 37I p.; t. 2: 457 p.; t. 3: 373 p. 4e édition -

Histoire du Canada depuis sa découverte jusqu'à nos jours, Montréal, C.O.

Beauchemin \& Fils, I882, 3 tomes; t. I: xxii, 397 p.; t. 2: 467 p.; t. 3: 407 p. Un quatrième volume s'ajoute à cette édition: Histoire du Canada depuis sa découverte jusqu'à nos jours. Notice biographique par M. Chauveau et Table analytique par M. Benjamin Sulte, précédées de 'Notre Histoire', poésie de M. Louis Fréchette, Montréal, C.O. Beauchemin \& Fils, 1883, I4, cccxcviii p. Ce titre sur la couverture est ambigu. A la page is est imprimé: François-Xavier Garneau, sa vie et ses oeuvres. Il s'agit en effet d'une étude sur la vie et l'oeuvre de Garneau qui, à proprement parler, ne devrait pas être comptée comme une partie de l'Histoire du Canada, mais comme une étude sur la vie et l'oeuvre de l'auteur, en tête du premier tome de la quatrième édition de l'Histoire du Canada. Selon l'entente avec l'éditeur, J.-P.-O. Chauveau enregistra son ouvrage comme volume séparé. Celui-ci contient aussi le 'Discours prononcé sur la tombe de F.X. Garneau' dit par Chauveau, le is septembre I867, p. cclxvii-cclxxvi. (La quatrième édition de l'Histoire du Canada fut préparée par Alfred Garneau, fils aîné de l'historien.)

se édition -

Histoire du Canada, cinquième édition, revue, annotée et publiée avec une introduction et des appendices par son petit-fils Hector Garneau, Paris, Librairie Félix Alcan, 2 tomes; t. I: 1913, lvi, 607-610 p.; t. 2: 1920, xii, 744-748 p. Préface de Gabriel Hanotaux, membre de l'Académie française. Avec un portrait de Garneau.

6e édition -

Histoire du Canada, cinquième édition, revue, annotée et publiée avec une introduction et des appendices par son petit-fils Hector Garneau, Paris, Librairie Félix Alcan, 1920, 2 tomes; t. I: lviii, 606-608; t. 2: xii, 744-748. Préface de Gabriel Hanotaux. Avec un portrait de Garneau. Réimpression de la se édition revue et corrigée.

7 e édition -

Histoire du Canada, septième édition, revue, annotée et publiée avec un introduction et des appendices par son petit-fils Hector Garneau, Paris, Librairie Félix Alcan, 1928, 2 tomes; t. I: lviii, 606-609 p.; vol. 2: xii, 744-748 p. Avec un portrait de Garneau. (Réimpression de la sixième édition.) $8 \mathrm{e}$ édition -

Histoire du Canada, huitième édition entièrement revue et augmentée par son petit-fils, Hector Garneau, Montréal, Editions de l'Arbre, [1944-1946], 9 vol.; vol. I: 285 p. 'Nouvelle Introduction' par Hector Garneau, p. 7-17; vol. 2: 30I p.; vol. 3: 305 p.; vol. 4: 299 p.; vol. 5: 317 p.; vol. 6: 319 p.; vol. 7: 23I p.; vol. 8: 195 p.; vol. 9: 295 p. (Index alphabétique à partir de la p. 157.) (Edition du centenaire de l'Histoire du Canada.) 
ge édition -

Histoire du Canada français [sic], Montréal, Les Amis de l'Histoire, Copyright François de Beauval, Paris, 1969, 6 tomes; t. 1: 402 p.; t. 2: 344 p.; t. 3: 297 p.; t. 4: 305 p.; t. 5: 296 p.; t. 6: 35I p. (Sous un titre fautif, sans introduction ni notes, l'ouvrage reproduit la quatrième édition de l'Histoire du Canada. Nombreuses illustrations.)

2. 'Histoire du Canada' en traduction anglaise

History of Canada from the Time of its Discovery till the Union Year (1840-41). Translated from 'Histoire du Canada' of F.-X. Garneau, Esq. and Accompanied with Illustrations, Notes, by Andrew Bell, Montréal, John Lovell, I860, 3 vol.; vol. I: xxii, 404 p.; t. 2: 382 p.; t. 3: 442 p. Edition corrigée chez le même éditeur en I862; t. I: 556 p.; t. 2: 499 p. Réimpressions: en I866, par Richard Worthington de Montréal; en 1876, par Belford Brothers de Toronto.

3. Abrégés de l'Histoire du Canada'

Abrégé de l'Histoire du Canada, depuis sa découverte jusqu'à 1840, à l'usage des maisons d'éducation, Québec, A. Côté, 1856 , iv, 248, iv p.

Abrégé de l'Histoire du Canada, depuis sa découverte jusqu'à 1840, à l'usage des maisons d'éducation, Québec, A. Côté, 1858 , iv, 197, iii p. Nouvelle édition.

Abrégé de l'Histoire du Canada, depuis sa découverte jusqu'à 1840, à l'usage des maisons d'éducation, Montréal, Librairie canadienne de Fabre et Gravel,' I858, iv, 197, iii p. (Edition revue et corrigée par l'auteur.)

Abrégé de l'Histoire du Canada, depuis sa découverte jusqu'à 1840, Québec, A. Côté, 1865, iv, 197, iii p.

Abrégé de l'Histoire du Canada, depuis sa découverte jusqu'à 1840, Québec, A. Côté, 1865, iv, 197, iii p. (Avec une note sur la page de titre: 'Approuvé par le Conseil de l'Instruction publique du Bas-Canada'.)

Abrégé de l'Histoire du Canada, depuis sa découverte jusqu'à 1840, Montréal, Beauchemin et Valois, 1875, iv, 210, iv p: Nouvelle édition.

Abrégé de l'Histoire du Canada, depuis sa découverte iusqu'à 1840, Montréal, Beauchemin et Fils, I88I, iv, 214, iv p. (Approuvé par le Conseil de l'Instruction publique. Contient aussi la relation historique jusqu'en février I88I, grâce aux efforts conjoints d'Alfred Garneau et de P.-J.-O. Chauveau.)

III. Récit de voyage

Voyage en Angleterre et en France dans les années 1831, 1832 et 1833, dans le Journal de Québec, I2e année, $\mathrm{I} 854$, livraisons: $\mathrm{n}^{0} \mathrm{I} 33, \mathrm{I} 8$ nov.; $\mathrm{n}^{0} \mathrm{I} 35,28$ nov.; $\mathrm{n}^{0} \mathrm{I} 38,30$ nov.; $\mathrm{n}^{0} 146$, 19 déc.; $\mathrm{n}^{0} 148,23$ déc.; $\mathrm{n}^{0} 151$, 30 déc.; 13 e année, 1855 , livraisons: $\mathrm{n}^{0}$ 2, 4 janv.; $\mathrm{n}^{0} 5$, 13 janv.; $\mathrm{n}^{0} 7,18$ janv.; $\mathrm{n}^{0}$ IO, 25 janv.; $\mathrm{n}^{0}{ }^{13}$, rer févr.; $\mathrm{n}^{0}{ }^{17}$, Io févr.; $n^{0}$ I9, I5 févr.; $n^{0} 22,22$ févr.; $n^{0} 26,3$ mars; $n^{0} 31$, I5 mars; $n^{0} 34,22$ mars; $n^{0} 36$, 27 mars; $n^{0} 40,5$ avril; $n^{0} 43$, 12 avril; $n^{0} 47,21$ avril; $n^{0} 51$, I er mai; $n^{0} 53,5$ mai; $\mathrm{n}^{0} 55$, 10 mai; $\mathrm{n}^{0} 57$, I5 mai; $\mathrm{n}^{0} 60,24 \mathrm{mai}_{;} \mathrm{n}^{0} 62,29$ mai. Le texte paraît dans le coin intitulé: 'Le Feuilleton du Journal de Québec'.

Voyage en Angleterre et en France dans les années 1831, 1832 et 1833, Québec, Augustin Côté \& Cie, 1855, 252 p.

Voyage de Garneau, dans La Littérature canadienne de 1850-1860, Québec, Desbarats et Derbishire, I863, p. 179-257 (texte abrégé).

Voyages [sic], Québec, Léger Brousseau, 1878, 168 p. Cette édition reproduit le texte paru dans La Littérature canadienne de 1850-1860; réimpression en $188 \mathrm{I}$. 
Iv. Journaux et articles de journaux

I. Journaux

L'Abeille canadienne

Hebdomadaire. Fondé par Garneau et imprimé par Fréchette et Cie, 25, rue de la Montagne. Le premier numéro paraît le 7 décembre 1833 , le demier, le 8 février 1834 .

L'Institut ou Journal des étudiants

Hebdomadaire. Fondé par F.X. Garneau et l'avocat David Roy. Continue à sa façon le Journal des étudiants de Derome, et s'inspire des nouvelles idées culturelles de Vattemare. Le propriétaire-imprimeur est Joseph-V. de Lorimier du I8, rue Saint-Jean, Québec. Le journal compte douze livraisons: il paraît le 7 mars $184 \mathrm{I}$, il cesse de paraître le 22 mai 184 I.

2. Articles

Une cinquantaine d'articles publiés par Garneau sont consignés dans la 'Bibliographie critique' dans le cadre du projet Garneau. La plupart de ces écrits furent publiés dans la presse périodique de l'époque. Le début de ce genre de publication remonte au I5 février 1837 : Garneau commença alors à publier dans $L e$ Canadien ses 'Extraits historiques ...', une vingtaine en tout dont le demier parut le 25 août 1837 .

\section{v. Correspondance}

\section{Lettres de Garneau}

Les recherches dans le cadre du 'Projet Garneau' ont permis d'établir que nous possédons aujourd'hui 152 lettres de Garneau: autographes, copies, brouillons, fragments. C'est M. Marc Lebel qui est chargé d'en établir les textes et d'en faire une édition critique. Une liste de ces lettres a été publiée par M. Marc Lebel dans Bulletin du Centre de recherche en civilisation canadienne-française, $\mathrm{n}^{0} \mathrm{I} 6$, avril 1978 , p. 18-20.

\section{Lettres à Garneau}

Grâce aux recherches dans le cadre du 'Projet Garneau', nous avons recueilli quelque I 39 lettres; on en dressera prochainement la liste exacte.

vi. Avis de greffier

Il existe quelque quatre cents avis de greffier que Garneau avait rédigés et publiés dans les journaux de l'époque. Ils sont toujours de nature administrative. Nous en avons fait un relevé assez complet qui fait partie du 'Projet Garneau', section bibliographie.

\section{B. ETUDES SUR F.-X. GARNEAU}

Note bibliographique

Une bibliographie complète consacrée à la vie et à l'oeuvre de F.X. Garneau comprendrait facilement un volume; la note qui suit ne donne que quelques jalons bibliographiques. Suivant l'ordre chronologique, les données sont regroupées ici en quatre sections et concernent successivement la vie de Garneau, son Histoire du Canada, sa poésie et son Voyage.

I. Renseignements généraux sur la vie, l'époque et l'oeuvre de F.X. Garneau Casgrain, H[enri]-R[aymond], Un contemporain: F-X. Gameau, Québec, J.-N. Duquet, 1866, 135 p. Etude amorcée en 1862, publiée ensuite sous sa forme 
restreinte, première, dans le Courrier du Canada des 5 et 7 février I866, développée et parue par la suite dans La Foyer canadien, t. 4, 1866, p. 181-24ð’'.

Darveau, L[ouis]-M[ichel], Garneau, dans Nos hommes de lettres, Montréal, A.A. Stevenson, I873, p. 75-IOI.

[Chauveau, Pierre-Joseph-Olivier], François-Xavier Garneau, sa vie et ses oeuvres, par M. Chauveau, Montréal, Beauchemin et Valois, Librairies-Imprimeurs, 1883, cclxxxii p. A la fin du volume le 'Discours' de Chauveau, prononcé sur la tombe de Garneau, le I5 septembre I867, au cimetière de Belmont (p. cclxviicclxxvi).

Garneau, Hector, François-Xavier Garneau, dans le Bulletin du Parler français au Canada, vol. 9, I910-191 I, p. 214-226.

Bienville, Louise de (Mme Donat Brodeur), Figures et Paysages, Montréal, Beauchemin, 1931, v, 24I p., surtout: François-Xavier Garneau, p. 159-165.

Lanctot, Gustave, F.-X. Garneau, Toronto, The Ryerson Press, [1926], 197 p. Bibliographie: p. I77-185. Cet ouvrage fut réimprimé vingt ans plus tard: F.-X. Garneau, notre historien national, Montréal, Fides, 1946, 207 p. Bibliographie: p. I8I-195.

[Collectif], François-Xavier Gameau. Aspects littéraires de son oeuvre, Ottawa, Editions de l'Université d'Ottawa, 1966, 208 p. 'Visage des lettres canadiennes'. Ouvrage préparé sous la direction de Paul Wyczynski en collaboration avec Odette Condemine, François Gallays, Charles Bolduc et la Soeur Paul du Sauveur.

Wyczynski, Paul, Généalogie et chronologie de F.-X. Garneau, dans François-Xavier Garneau, Voyage en Angleterre et en France dans les années 1831, 1832 et 1833, Ottawa, 1968, p. II-30. Edition critique. Texte établi, annoté et présenté par Paul Wyczynski. Dans le même volume: une Bibliographie (oeuvre de Garneau et études sur Garneau), p. 3I-53.

Hamel, Réginald, John Hare et Paul Wyczynski, Garneau, François-Xavier Garneau, dans Dictionnaire pratique des auteurs québécois, Montréal, Fides, 1976, p. 273-277.

Savard, Pierre et Paul Wyczynski, Garneau, François-Xavier, dans Dictionnaire biographique du Canada, Québec, Les Presses de l'Université Laval - Toronto, University of Toronto Press, 1977, p. 327-335. (Esquisse biographique suivie d'une note bibliographique.)

[Savard, Pierre et Paul Wyczynski], François-Xavier Garneau, 1809-1866, Ottawa, Bibliothèque nationale du Canada, 1977, 160 p. Préface de Guy Sylvestre. Catalogue en français et en anglais de $1^{\prime}$ Exposition Garneau, tenue à la Bibliothèque nationale du Canada en octobre, novembre et décembre 1978, ensuite au Centre culturel canadien de Paris (décembre I978) et aux universités de Grenoble et de Bordeaux, en 1979.

Cantin, Pierre, Normand Harrington et Jean-Paul Hudon, Garneau, FrançoisXavier, dans Bibliographie de la critique de la littérature québécoise dans les revues des xixe et xxe siècles, Ottawa, Centre de recherche en civilisation canadienne-française, 1979, t. 3, articles 8777-8821. 'Documents de travail du Centre de recherche en civilisation canadienne-française', ${ }_{4} 4$.

II. Renseignements sur l'Histoire du Canada de F.-X. Garneau

Le Moine, J[ames]-M[acPherson], Nos quatre historiens modernes, Bibaud, Garneau, Ferland, Faillon, dans Mémoires de la Société royale du Canada pour les 
années 1882 et 1883, Montréal, Dawson frères, I883, sect. I, p. I-6.

Casgrain, H[enri]-R[aymond], F.-X. Garneau et Francis Parkman, Montréal, Beauchemin, I912, I39 p.

Garneau, Hector, Introduction dans François-Xavier Garneau, Histoire du Canada, Paris, Librairie Félix Alcan, I9I 3, se édition, p. xxv-xli.

Arles, Henri d' (Henri Beaudé), Nos historiens, Montréal, Bibliothèque de l'Action française, 1921, 243 p.; surtout p. 83-123.

Robitaille, Georges, Etudes sur Garneau, critique historique, Montréal, Librairie d'Action canadienne-française, I929, $253 \mathrm{p}$.

Chapais, Thomas, Discours et conférences, Québec, Librairie Garneau, I935, 5 Io p., 'Garneau', p. 345-380.

Garneau, Hector, Nouvelle Introduction à l'Histoire du Canada de F.-X. Garneau, dans Le Canada français, vol. 30, $\mathrm{n}^{0} 7$, mars 1943, p. 48I-485.

Frégault, Guy, Actualité de Garneau, dans l'Action universitaire, mars I945, p. 8I6.

[Collectif], Centenaire de l'Histoire du Canada de François-Xavier Garneau. Deuxième semaine d'histoire à l'Université de Montréal, 23-27 avril 1945, Montréal, Société historique de Montréal, 1945, 460 p. Ouvrage préparé sous la direction de Jean-Jacques Lefebvre. On y trouve les études de Gustave Lanctot, de Lionel Groulx, d'Olivier Maurault, de Jean Bruchési, d'Armand Yon, de Thomas Charland, de Georges Robitaille, de Maurice Hébert.

Id., Histoire du Canada, dans The Canadian Historical Review, vol. $27, \mathrm{n}^{0} 3$, septembre 1946, p. 3II-315.

Bouchard, f.s.g., frère Amédée-Joseph-Lucien, 'L'Histoire du Canada de Garneau: étude comparée de la première et de la cinquième éditions', thèse de maîtrise, Université de Montréal, 1953, vi, 134 p.

Saint-Martin, Louis-Philippe, L'Histoire du Canada de F.-X. Garneau et la critique, dans Revue d'histoire de l'Amérique française, vol. 8, $\mathrm{n}^{0} \mathrm{I}$, juin $1954, \mathrm{p} .380-394$.

Hathorn, Ramon J., Garneau, disciple de Thierry, dans Mosaïc, vol. I, $\mathrm{n}^{0} \mathrm{I}$, octobre I967, p. 66-78.

Barette, m.i.c., soeur Georgette, 'Garneau et la critique', thèse de maîtrise, Université d'Ottawa, I98, I32 p. Présentation des études et articles consacrés aux trois premières éditions de l'Histoire du Canada.

Pritchard, James-S., Some Aspects of the Thought of F.-X. Garneau, dans The Canadian Historical Review, vol. 51, $\mathrm{n}^{0}$ 3, sept. 1970, p. 276-291.

Van Rutten, Pierre-M., Le Paradoxe du style de F-X. Garneau, dans Revue de l'Université d'Ottawa, vol. 3, $\mathrm{n}^{0}$ 2, avril 1973, p. 15-23.

Lebel, Marc, Garneau disciple de Michelet, dans Le Bulletin de Centre de recherche en civilisation canadienne-française, $\mathrm{n}^{0}$ 9, décembre 1974, p. I-4.

Savard, Pierre, Les Rééditions de l'Histoire du Canada de F.X. Garneau, dans Revue d'histoire de l'Amérique française, vol. $28, \mathrm{n}^{0}{ }_{4}$, mars 1975, p. 539-553.

Savard, Pierre et Paul Wyczynski, Histoire du Canada depuis sa découverte jusqu'à nos jours de François-Xavier Garneau dans Dictionnaire des oeuvres littéraires du Québec, Montréal, Fides, 1978, t. I, p. 347-355.

III. Renseignements sur la poésie de F.X. Garneau

Chauveau, P.-J.-O., Etude sur les commencements de la poésie française au Canada et en particulier sur les poésies de M. F.-X. Garneau, dans Mémoires de la Société royale du Canada, 1882-1883, Montréal, Dawson frères, I883, I e série, section I, 
p. 65-84. Etude lue par Chauveau, le 26 mai 1883 , à la réunion de la Société royale du Canada, à Ottawa.

Bisson, Laurence-A., Le Romantisme littéraire au Canada, Paris, Droz, 1932, 285 p.; surtout p. 57-77.

Condemine, Odette, François-Xavier Gameau, poète, dans François-Xavier Garneau. Aspects littéraires de son oeuvre, Ottawa, Editions de l'Université d'Ottawa, 1966, p. 9-43.

Lortie, s.c.o., Jeanne d'Arc, La Poésie nationaliste au Canada français (1606-1867), Québec, Les Presses de 1'Université Laval, 1975, ix, 535 p.; surtout p. 250-27I, passim. 'Vie des lettres québécoises'.

Wyczynski, Paul, [Poèmes épars] de François-Xavier Garneau, dans Dictionnaire des oeuvres littéraires du Québec, Montréal, Fides, 1978, vol. I, p. 582-585.

Iv. Renseignements sur le Voyage en Angleterre et en France dans les années I831, 1832 et 1833

Roy, P[ierre]-G[eorges], Voyage en Angleterre et en France, dans Bulletin des recherches historiques, Lévis, vol. $4, \mathrm{n}^{0}$ 9, sept. 1898, p. 279-281 .

Mailhot, l'abbé Charles -E., Le 'Voyage en Angleterre et en France' de F.-X. Garneau, dans Bulletin des recherches historiques, Lévis, vol. 4, nº 6, 1898, p. 190.

[Wyczynski, Paul], François-Xavier Garneau, Voyage en Angleterre et en France dans les années 1831,1832 et 1833, Ottawa, Editions de l'Université d'Ottawa, I968, 377 p. (Edition critique.) 
